\title{
Why Should We Integrate Biomarkers into Complex Trials?
}

\author{
Frauke Musial \\ National Research Centre in Complementary and Alternative Medicine, NAFKAM, Department of Community Medicine, \\ Faculty of Health Science, University of Troms $\varnothing$, Norway
}

Research in complementary and alternative medicine (CAM) encounters a variety of challenges, such as potentially synergistic, multimodal, and complex interactions, aiming at behavioral changes in the patient with long-term effects. Furthermore, these interventions are often dependent on the relationship between practitioner and patient as well as on patients' preferences, expectations, and motivations [1]. Moreover, patients seeking CAM care often suffer from chronic diseases and multiple pathologies [1]. The challenges these complex and multifactorial clinical settings impose to clinical research are even potentiated when it comes to whole medical systems, such as traditional medical systems (e.g. Ayurveda) or homeopathy, whose traditions not only include particular treatment modalities but also diagnosis and patientpractitioner interactions as well as techniques for changing the patient's behavior [2,3]. Moreover and almost as a general rule, research in CAM faces the challenge that treatment is not derived from a biological hypothesis as it is (ideally) the case in pharmacological trials. CAM research faces the fact that the therapies are practiced regardless of whether the biological mechanisms, comparative effectiveness, component efficacy, or even safety aspects are known [4].

All these preconditions, the complexity of the interventions and the treatment setting, the fact that many of the patients often suffer from several chronic diseases, and the fact that there is generally no clear hypothesis on the biological mechanism of action make the applicability of the gold standard in clinical research, the randomized controlled trial $(\mathrm{RCT})$, questionable $[1,2,4,5]$. In the field of CAM, there has been a growing understanding and agreement that not more RCTs but the development of appropriate methodological and statistical tools for the investigation of complex interventions is the answer to the quest $[1,2,4]$. But that also includes in part a re-discussion and re-definition of the 'outcomes' concept [5].

\section{KARGER \\ Fax +497614520714 \\ Information@Karger.de}

www.karger.com
(C) 2012 S. Karger GmbH, Freiburg $1661-4119 / 12 / 0195-0232 \$ 38.00 / 0$

Accessible online at:

www.karger.com/fok
As Paterson et al. [5] consequently emphasized, outcomes which are appropriate for complex health interventions ideally are able to detect changes and dynamics. In complex health interventions we often see that a treatment, even under strictly controlled conditions, shows its effect in the way a subject adapts to a challenge. As Paterson et al. [5] pointed out, a process is something that enables the individual to adapt to varying experiences. In conclusion, the change of process, not of status, may be the indicator of improved health. However, the related outcomes are complex, they often represent time series, and there is a substantial need for new methodological and statistical approaches $[2,5]$.

These challenges may even be enhanced when trying to integrate biomarkers into complex interventional trials, since biomarkers are usually identified and selected on the basis of a clear, biological hypothesis investigated in a design which allows for the application of the 'principle of isolated variation' in experimentation. The principle of isolated variation requires that the treatment groups are varied according to only one particular variable. The classical pharmacological RCT is a typical example in that it only varies the content of the active drug against a placebo.

With regard to biomarkers and the 'principle of isolated variation', complex trials in the CAM field struggle with two major challenges:

a) The mechanism of action of the intervention is often unclear, and the explanatory models are rarely grounded in physiology/pathophysiology.

b) The interventions are complex and often address several aspects of the patient and his or her symptoms, which from an understanding of a specific pathophysiology may be seen as unrelated to the disease or at least only relevant in second or third line.

In the light of the complexity of the problem, can we integrate biomarkers into these multidimensional trials and is there a 
need to do so? The answer is clearly 'yes' $[2,5]$. There is even an explicit call for 'objective change indicators' which should preferably be biomarkers [5].

But how can biomarkers be selected rationally so that they can be utilized as 'objective change indicators'? The challenge can be addressed through the implementation of two general principles which refer a) to the process of selecting the appropriate biomarkers and b) the application of small 'sub-experiments' embedded in a complex trial:

a) The guided selection of biomarkers should be driven by the physiological system where the treatment-induced change can be expected, such as the CNS, the autonomic nervous system, etc. Moreover, the level where the change is to be expected needs to be taken into account (e.g. for the CNS: at the level of the brain or below?) [6]. The most rational guidance is provided by the relevant symptoms of the patient. If this is e.g. pain, then a pain-related biomarker is appropriate.

b) 'Sub-experiments' or challenges to the system are needed when complex interventions cannot be expected to alter biomarkers at a resting level/state, but may alter the physiological response to psychophysiological challenge (e.g. mental stress, physical activity). In these sub-experiments, specific challenges to the physiological system in focus, e.g. a stress test, is applied and the change in response to the challenge (e.g. change in heart rate variability) is measured as outcome.

Heart rate variability as a complex time series and associated variability analysis techniques [7] or multivariate approaches [8] are a good example for the availability of the advanced methodology needed. As for pain-related interventions, the quantitative sensory testing system provides a biomarker, directly targeting the pain processing pathways and networks, which includes a series of tests applying challenges e.g. in the form of controlled pain stimuli to the pain processing system $[9,10]$.

Nonetheless, integrating biomarkers in complex CAM trials is logistically challenging, tedious, time-consuming, and costly. Why should we do it? Because the CAM field struggles with the fact that the interventions are often applied for centuries, that the clinical effectiveness of some is known and is sometimes even striking, but that almost as a general rule, there is no good understanding why they work. Why is that so important? Because if CAM procedures are to be developed further, if we want to make them better, if we want to tailor them even more to the complexity of the individual patient's needs, then knowledge of the psychophysiology involved in a healing process is needed as much as knowledge of the involved context factors of these complex interventions.

\section{References}

1 MacPherson H, Peters D, Zollman C: Closing the evidence gap in integrative medicine. BMJ 2009; 339:b3335.

2 Ritenbaugh C, Aickin M, Bradley R, Caspi O, Grimsgaard S, Musial F: Whole systems research becomes real: new results and next steps. J Altern Complement Med 2010;16:131-137.

$\checkmark 3$ Kessler C, Michalsen A: The role of whole medical systems in global medicine. Forsch Komplementmed 2012;19:65-66.

$\checkmark 4$ Fønneb $\varnothing$ V, Grimsgaard S, Walach H, Ritenbaugh C, Norheim AJ, MacPherson H, Lewith G, Launs $\varnothing$ L, Koithan M, Falkenberg T, Boon H, Aickin M: Researching complementary and alternative treatments - the gatekeepers are not at home. BMC Med Res Methodol 2007;7:7.
5 Paterson C, Baarts C, Launs $\varnothing$ L, Verhoef MJ: Evaluating complex health interventions: a critical analysis of the 'outcomes' concept. BMC Complement Altern Med 2009;9:18.

6 Musial F, Michalsen A, Dobos G: Functional chronic pain syndromes and naturopathic treatments: neurobiological foundations. Forsch Komplementmed 2008;15:97-103.

7 Bravi A, Longtin A, Seely AJ: Review and classification of variability analysis techniques with clinical applications. Biomed Eng Online 2011;10:90. .

8 Thayer JF, Ahs F, Fredrikson M, Sollers JJ 3rd, Wager TD: A meta-analysis of heart rate variability and neuroimaging studies: implications for heart rate variability as a marker of stress and health. Neurosci Biobehav Rev 2012;36:747-756.
9 Rolke R, Baron R, Maier C, Tölle TR, Treede RD Beyer A, Binder A, Birbaumer N, Birklein F, Bötefür IC, Braune S, Flor H, Huge V, Klug R, Landwehrmeyer GB, Magerl W, Maihöfner C, Rolko C, Schaub C, Scherens A, Sprenger T, Valet M, Wasserka B: Quantitative sensory testing in the German Research Network on Neuropathic Pain (DFNS): standardized protocol and reference values. Pain 2006;123:231-243. Erratum in: Pain 2006; 125:197.

10 Rolke R, Magerl W, Campbell KA, Schalber C, Caspari S, Birklein F, Treede RD: Quantitative sensory testing: a comprehensive protocol for clinical trials. Eur J Pain 2006;10:77-88. 